\title{
Higgs Inflation With Four-form Couplings
}

\author{
C.J. Ouseph ${ }^{a, b}$, Kingman Cheung ${ }^{a, b, c, d}$ \\ a Department of Physics, National Tsing Hua University, Hsinchu 300, Taiwan \\ ${ }^{b}$ Institute of Astronomy, National Tsing Hua University, Hsinchu 300, Taiwan \\ c Physics Division, National Center for Theoretical Sciences, Hsinchu 300, Taiwan \\ $d$ Division of Quantum Phases and Devices, School of Physics, \\ Konkuk University, Seoul 143-701, Republic of Korea
}

(Dated: March 9, 2020)

\begin{abstract}
We consider a new inflationary model in which an antisymmetric tensor field $A_{\nu \rho \sigma}$ and its fourform field strength $F_{\mu \nu \rho \sigma}=4 \partial_{[\mu} A_{\nu \rho \sigma]}$ are coupled to the scalar sector of the standard model and to the Ricci scalar $\mathcal{R}$. The four-form field induces modifications to the Higgs self-coupling constant, the cosmological constant, and the non-minimal coupling constant, which results in the modification to the inflaton potential. We also show that there is no need for the Higgs-gravity coupling in the presence of four-form-gravity interaction, but still can produce the right amount of density perturbation for inflation.
\end{abstract}




\section{INTRODUCTION}

Recently, an interesting proposal was put forward to give a simultaneous solution to the smallness of both cosmological constant and the Higgs vacuum expectation value (VEV) [1, 2]. This is based on the argument that if the Higgs VEV is explained by a choice in cosmological selection among the landscape, the cosmological-constant scale should also be addressed by the same selection.

The framework is the standard model (SM) plus a non-dynamical field described by a 4-form. The 4-form can arise from some nontrivial topological sectors. When the 4-form couples to gravity, it contributes to the vacuum energy. If it couples to the Higgs field at the same time, it can generate a number of configurations for the Higgs VEV, so that the acceptable Higgs-boson VEV and mass can be selected. Simultaneously, the smallness of the Cosmological Constant can be selected. The coupling of the 4-form to gravity was also proposed to address the problem of inflation [3], and more recently in Refs. [4, 5].

Cosmological inflation is the most favorable theory of early universe. It not only explains the absence of a number of relics that should have existed from the Big Bang, but also provides the seeds for the growth of structures in the Universe. In the last two decades, people have been attempting to figure out the most promising candidate for cosmological inflation. There are plenty of attempts to address inflation within the framework of the standard model (SM) and theories beyond the SM. The SM Higgs field is always a fascinating candidate as the inflaton because of the non-requirement of additional scalar degree of freedom. However, the minimal Higgs inflation model is not favorable, if not ruled out, because of the fine-tuned value of the Higgs self-coupling constant $\lambda$. A non-minimal coupling between the SM Higgs field and the Ricci scalar $\mathcal{R}$ [6] was then introduced with the hope of relaxing the value of $\lambda$. However, such kind of attempts may lead to unitarity violation. In order to avoid the problem of unitary violation in Higgs sector, we study the scenario that the four-form field $F_{\mu \nu \rho \sigma}$ is coupled to the Higgs field and the Ricci scalar. Consequently, the Higgs self-coupling constant and the non-minimal coupling constant are modified. In addition to the cosmological constant $\Lambda$ being scanned to an effective value $\Lambda_{e f f}$, the Higgsboson mass $m_{g e n}$ can also be generated before the electroweak symmetry breaking [1, 2].

In this work, we consider two different inflationary models of non-minimal couplings. The first one is the more general model of inflation, in which both the SM Higgs and the four- 
form fields couple to gravity and it results in an effective non-minimal coupling constant $\xi_{\text {eff }}$ in the model. In the second model, only the non-minimal coupling of $F_{\mu \nu \rho \sigma}$ with gravity is considered. The SM Higgs is free from the non-minimal coupling, because of non-necessity of Higgs-gravity coupling for inflation. We then compare the results of the minimal and non-minimal Higgs inflation models, with respect to the most recent values of spectral index and the tensor-to-scalar ratio.

The organization is as follows. In Sec. II, we describe the non-minimal coupling models. In Sec. III, we calculate the spectral index and tensor-to-scalar ratio of the models. Section III A and B deal with the comparison between the minimal and non-minimal coupling models. We conclude in Sec. IV.

\section{THE 4-FORM INTERACTIONS}

Let us first consider an inflationary model in which the SM Higgs field is coupled to the scalar curvature $\mathcal{R}$, to the tensor 3 -form field $A_{\nu \rho \sigma}$, and to its four-form field strength $F_{\mu \nu \rho \sigma}=4 \partial_{[\mu} A_{\nu \rho \sigma]}$ in a non-minimal way. Here we are writing the Higgs field $H=h / \sqrt{2}$ in the unitary gauge without specifying the term with the VEV. The Lagrangian for the Higgs field $h$ is given by

$$
\mathcal{L}=\mathcal{L}_{0}+\mathcal{L}_{\text {int }}+\mathcal{L}_{s}+\mathcal{L}_{L}+\mathcal{L}_{\text {memb }}
$$

where

$$
\begin{array}{r}
\mathcal{L}_{0}=\sqrt{-g}\left[M_{\mathrm{PL}}^{2} \frac{\mathcal{R}}{2}+\frac{b^{2} \mathcal{R}^{2}}{2}-\frac{1}{48} F^{\mu \nu \rho \sigma} F_{\mu \nu \rho \sigma}-\frac{1}{2}\left(\partial_{\mu} h\right)^{2}-\frac{\lambda}{4} h^{4}-\Lambda\right] \\
\mathcal{L}_{i n t}=\frac{C_{1}}{48} \epsilon^{\mu \nu \rho \sigma} F_{\mu \nu \rho \sigma} h^{2}+\frac{1}{2} \xi_{1} h^{2} \mathcal{R}+\xi_{2} \epsilon^{\mu \nu \rho \sigma} F_{\mu \nu \rho \sigma} \mathcal{R} \\
\mathcal{L}_{s}=\frac{1}{6} \partial_{\mu}\left[\left(\sqrt{-g} F^{\mu \nu \rho \sigma}-C_{1} \epsilon^{\mu \nu \rho \sigma} h^{2}+\epsilon^{\mu \nu \rho \sigma} \xi_{2} \mathcal{R}\right) A_{\nu \rho \sigma}\right] \\
\mathcal{L}_{L}=\frac{q_{1}}{24} \epsilon^{\mu \nu \rho \sigma}\left[F_{\mu \nu \rho \sigma}-4 \partial_{[\mu} A_{\nu \rho \sigma]}\right] \\
\mathcal{L}_{m e m b}=\frac{q_{2}}{2} \int d^{3} \zeta\left[\delta^{4}(x-x(\zeta)) A_{\nu \rho \sigma} \frac{\partial x^{\nu}}{\partial \zeta^{a}} \frac{\partial x^{\rho}}{\partial \zeta^{b}} \frac{\partial x^{\sigma}}{\partial \zeta^{c}} \epsilon^{a b c}\right]
\end{array}
$$

We are considering the same form of Lagrangian as in Refs. [4, 5]. We have introduced the terms $\xi_{1} h^{2} \mathcal{R}$ and $\xi_{2} \epsilon^{\mu \nu \rho \sigma} F_{\mu \nu \rho \sigma} \mathcal{R}$ in the $\mathcal{L}_{\text {int }}$, where $\xi_{1}$ is the non-minimal coupling of Higgs

field with gravity and the four-form field is coupled to the Ricci scalar via $\xi_{2}$. The $\Lambda$ is the cosmological constant. The required Lagrangian for the inflationary scenario obtained after 
integrating out the $F_{\mu \nu \rho \sigma}$ field can be rewritten as,

$$
\begin{array}{r}
\mathcal{L}=\sqrt{-g}\left[M_{\mathrm{PL}}^{2} \frac{\mathcal{R}}{2}+\frac{b^{2} \mathcal{R}^{2}}{2}-\frac{1}{2}\left(\partial_{\mu} h\right)^{2}-\frac{\lambda}{4} h^{4}-\Lambda+\right. \\
\left.\frac{1}{2} \xi_{1} h^{2} \mathcal{R}-\frac{1}{2}\left(C_{1} h^{2}-\xi_{2} \mathcal{R}+q_{1}\right)^{2}-\frac{1}{6} \epsilon^{\mu \nu \rho \sigma} \partial_{\mu} q_{1} A_{\nu \rho \sigma}+\mathcal{L}_{m e m b}\right] .
\end{array}
$$

From the equation of motion of $A_{\nu \rho \sigma}$,

$$
\epsilon^{\mu \nu \rho \sigma} \partial_{\mu} q_{1}=\frac{q_{2}}{2} \int d^{3} \zeta\left[\delta^{4}(x-x(\zeta)) \frac{\partial x^{\nu}}{\partial \zeta^{a}} \frac{\partial x^{\rho}}{\partial \zeta^{b}} \frac{\partial x^{\sigma}}{\partial \zeta^{c}} \epsilon^{a b c}\right]
$$

thus $q_{1}$ get quantized by $q_{1}=n q_{2}$, where $n$ is an integer.

Other than the non-minimal couplings of Higgs field with gravity and the 4-form field with the gravity, there is also the $\mathcal{R}^{2}$ coupling. In order to simplify the Lagrangian we perform a dual transformation of the $\mathcal{R}^{2}$ term in Eq. (7) in terms of a real scalar $\psi, \psi=$ $\mathcal{R} \sqrt{b^{2}-\xi_{2}^{2}} \times \frac{(1 \pm i \sqrt{3})}{2}$, and we obtain

$$
\mathcal{L}=\sqrt{-g}\left[\frac{\Omega(h, \psi, q)}{2} \mathcal{R}-\frac{1}{2}\left(\partial_{\mu} h\right)^{2}-\Lambda-\frac{q_{1}^{2}}{2}-h^{4}\left(\frac{\lambda}{4}+\frac{C_{1}^{2}}{2}\right)-\frac{1}{2} h^{2}\left(2 q_{1} c_{1}\right)-\frac{1}{2} \psi^{2}\right]
$$

with

$$
\Omega(h, \psi, q)=\left[\left(M_{\mathrm{PL}}^{2}+h^{2}\left(\xi_{1}+2 C_{1} \xi_{2}\right)+2 \xi_{2} q_{1}\right)+\sqrt{b^{2}-\xi_{2}^{2}} \psi\right] .
$$

Here we make a field redefinition:

$$
\sigma=\left(\xi_{1}+2 C_{1} \xi_{2}\right) \frac{h^{2}}{\xi_{2}}+2 q_{1}+\frac{\sqrt{b^{2}-\xi_{2}^{2}}}{\xi_{2}} \psi
$$

and Eq. (9) then becomes

$$
\begin{array}{r}
\mathcal{L}=\sqrt{-g}\left[\frac{1}{2}\left(M_{\mathrm{PL}}^{2}+\xi_{2} \sigma\right) \mathcal{R}-\frac{1}{2}\left(\partial_{\mu} h\right)^{2}-\Lambda-\frac{q_{1}^{2}}{2}-\right. \\
\left.h^{4}\left(\frac{\lambda}{4}+\frac{C_{1}^{2}}{2}\right)-\frac{1}{2} h^{2}\left(2 q_{1} c_{1}\right)-\frac{1}{2} \frac{\xi_{2}^{2}}{b^{2}-\xi_{2}^{2}}\left(\sigma-\left(\frac{h^{2}}{\xi_{2}}\left(\xi_{1}+2 \xi_{2} C_{1}\right)+2 q_{1}\right)\right)^{2}\right] .
\end{array}
$$

The potential of the Lagrangian can be expanded as

$$
V(h, q, \sigma)=h^{4} \lambda_{e f f}+m_{g e n}^{2} h^{2}+\Lambda+\frac{q_{1}^{2}}{2}(1-4 \beta)-2 q_{1} \beta \sigma+\beta \frac{\sigma^{2}}{2},
$$

where $\beta=\frac{\xi_{2}^{2}}{b^{2}-\xi_{2}^{2}}$, and $\lambda_{e f f}$ defined as,

$$
\lambda_{e f f}=\left(\frac{\lambda}{4}+\frac{C_{1}^{2}}{2}+\frac{\beta}{2}\left(\frac{\xi_{1}}{\xi_{2}^{2}}+\frac{2 C_{1}}{\xi_{2}}\right)^{2}\right) .
$$


The modified Cosmological Constant becomes

$$
\Lambda_{\mathrm{eff}}=\Lambda+\frac{q_{1}^{2}}{2}(1-4 \beta)
$$

The generated Higgs mass from the 4-form interaction can be obtained as

$$
m_{\mathrm{gen}}^{2}=h^{2}\left[c_{1} q_{1}+\beta\left(\frac{\xi_{1}}{\xi_{2}^{2}}+\frac{2 C_{1}}{\xi_{2}}\right)\left(2 q_{1}-\sigma\right)\right] .
$$

It was shown that desirable values for Higgs mass and cosmological constant can be achieved with scanning [1, 2].

\section{COSMOLOGICAL INFLATION AND DENSITY FLUCTUATION}

A lot of works in literature have discussed about the SM Higgs field as the inflaton. Here we introduce a new scenario in which we describe how the Higgs-gravity coupling and the coupling between Higgs and the 4-form flux can give rise to successful inflation. The Lagrangian in Jordan frame can be described as follows

$$
\mathcal{S}_{J}=\int d^{4} x \sqrt{-g}\left(\frac{1}{2}\left(M_{\mathrm{PL}}^{2}+\xi_{2} \sigma\right) \mathcal{R}+\frac{1}{2}\left(\partial_{\mu} h\right)^{2}-V\left(h, q_{1}, \sigma\right)\right) .
$$

We can integrate out the $\sigma$ field from the $\mathrm{Eq}(12) \sigma=\left(\xi_{1}+2 C_{1} \xi_{2}\right) \frac{h^{2}}{\xi_{2}}+2 q_{1}$. However, some components of the the generated Higgs mass $m_{g e n}$ and the $\lambda_{\text {eff }}$ disappear after integrating out the $\sigma$ field from the Lagrangian.

$$
\begin{aligned}
& \lambda_{\text {eff }}=\frac{\lambda}{4}+\frac{C_{1}^{2}}{2} \\
& m_{\text {gen }}^{2}=h^{2} c_{1} q_{1} .
\end{aligned}
$$

In this inflation scenario, we only consider the highest power of the Higgs field, the $h^{4}$, as the inflaton in the potential, i.e., we are neglecting the $h^{2}$ term. We can get rid of the non-minimal coupling of gravity from the Lagrangian using the conformal transformation [7]. Here we are performing the conformal transformation from the Jordan frame to the Einstein frame:

$$
\hat{g}_{\mu \nu}=\Omega g_{\mu \nu}, \quad \Omega=\left(1+\frac{h^{2} \xi_{e f f}+2 \xi_{2} q_{1}}{M_{\mathrm{PL}}^{2}}\right), \text { with } \xi_{\mathrm{eff}}=\xi_{1}+2 \mathrm{C}_{1} \xi_{2} .
$$

The conformal transformation gives rise to a non-minimal kinetic term for the inflaton field. We redefined the field as

$$
\frac{d \phi}{d h}=M_{\mathrm{PL}} \sqrt{\frac{\Omega}{\Omega^{2}}+\frac{3}{2} \frac{\left(\frac{d \Omega}{d h}\right)^{2}}{\Omega^{2}}}
$$


with

$$
\phi=\sqrt{\frac{3}{2}} M_{\mathrm{PL}} \ln \Omega
$$

The action in the Einstein frame is defined as

$$
\mathcal{S}_{E}=\int d^{4} x \sqrt{-\hat{g}}\left(\frac{M_{\mathrm{PL}}^{2} \hat{\mathcal{R}}}{2}+\frac{1}{2} \partial_{\mu} \phi \partial^{\mu} \phi-U(\phi)\right)
$$

where $U(\phi)=\frac{V\left(h, q_{1}\right)}{\Omega^{2}}$ with $V\left(h, q_{1}\right)=\lambda_{e f f} h^{4}$. The flat exponential inflaton potential can be expressed as

$$
U(\phi)=\frac{M_{\mathrm{PL}}^{4} \lambda_{e f f}}{\xi_{\text {eff }}^{2}}\left[\frac{\left(\exp \left(\sqrt{\frac{2}{3}} \frac{\phi}{M_{\mathrm{PL}}}\right)-\left(1+\frac{2 \xi_{2} q_{1}}{M_{\mathrm{PL}}^{2}}\right)\right)^{2}}{\exp \left(2 \sqrt{\frac{2}{3}} \frac{\phi}{M_{\mathrm{PL}}}\right)}\right]
$$

with $\lambda_{\text {eff }}=\frac{\lambda}{4}+\frac{C_{1}^{2}}{2}$.

The slow-roll parameters can be calculated analytically as a function of $h(\phi)$ :

$$
\begin{aligned}
& \epsilon=\frac{M_{\mathrm{PL}}^{2}}{2}\left(\frac{\frac{\partial U}{\partial \phi}}{U}\right)^{2}=\frac{4\left(M_{\mathrm{PL}}^{2}+2 \xi_{2} q_{1}\right)^{2}}{3 \xi_{\text {eff }}^{2} h^{4}} \\
& \eta=M_{\mathrm{PL}}^{2}\left(\frac{\frac{\partial^{2} U}{\partial \phi^{2}}}{U}\right)=\left(\frac{4}{3}\right) \frac{\left(M_{\mathrm{PL}}^{2}+2 \xi_{2} q_{1}\right)\left(M_{\mathrm{PL}}^{2}+2 \xi_{2} q_{1}-\xi_{\text {eff }} h^{2}\right)}{\xi_{\text {eff }}^{2} h^{4}}
\end{aligned}
$$

Note that $\epsilon=1$ at the end of slow roll. The field value of inflaton at the end of inflation is given by $h_{\text {end }}=\left(\frac{4}{3}\right)^{\frac{1}{4}} M_{\mathrm{PL}} \sqrt{\frac{\left(1+\frac{2 \xi_{2} q_{1}}{M_{\mathrm{PL}}^{2}}\right)}{\xi_{e f f}}}$. The number of $e$-foldings can be calculated as

$$
\mathcal{N}=\int_{h_{\text {end }}}^{h_{0}} \frac{1}{M_{\mathrm{PL}}^{2}}\left(\frac{U}{\frac{\partial U}{\partial h}}\left(\frac{\partial \phi}{\partial h}\right)^{2}\right) d h
$$

For $\mathcal{N}=60$ the value of $h_{0}=9.00859 \times M_{\mathrm{PL}} \sqrt{\frac{\left(1+\frac{2 \xi_{2} q_{1}}{M_{\mathrm{PL}}^{2}}\right)}{\xi_{e f f}}}$. We can constrain the value of $\lambda_{\text {eff }}$ and $\xi_{\text {eff }}$ with the COBE normalization $\frac{U}{\epsilon}=\left(0.027 M_{\mathrm{PL}}\right)^{4}$. Now the value of $\frac{\lambda_{e f f}}{\xi_{\text {eff }}^{2}}=$ $1.10256 \times 10^{-10}$. The spectral index $n_{s}=1-6 \epsilon+2 \eta$ and the tensor-to-scalar ratio $r=16 \epsilon$ for $\mathcal{N}=60$ is calculated to be $n_{s}=0.9633, r=0.0032$. The predicted values for $n_{S}$ and $r$ are in excellent agreement with the Planck 2018 data within $1 \sigma$ [8]. The reheating era started at the end of the slow roll. The SM Higgs field would start to interact with other SM particles at the time of reheating, $T_{r e h} \simeq\left(\frac{2 \lambda_{\text {eff }}}{\pi^{2} g^{*}}\right)^{\frac{1}{4}} \frac{M_{\mathrm{PL}}}{\sqrt{\xi_{\text {eff }}}} \simeq 2 \times 10^{15} \mathrm{GeV}$, where $g^{*}$ is the number of degrees of freedom of the SM at the time of reheating and $g^{*}=106.75$. 


\section{A. Realization of Non-Minimal Higgs Inflation with $\xi_{2}=0$ limit}

By switching off $\xi_{2}\left(\xi_{2}=0\right)$ we can reproduce the same form of inflaton potential of Bezrukov and Shaposhnikov's [6] with $\lambda_{\text {eff }}=\frac{\lambda}{4}+\frac{C_{1}^{2}}{2}$ (by switching off $C_{1}\left(C_{1}=0\right.$ ), the entire model become the non-minimal Higgs inflation):

$$
U_{E}=\frac{\lambda_{e f f} M_{\mathrm{PL}}^{4}}{\xi^{2}}\left(1-\exp \left(-\frac{2 \phi}{\sqrt{6} M_{\mathrm{PL}}}\right)\right)^{2}
$$

where $\xi=\xi_{1}$, the Higgs-gravity coupling. The slow-roll parameters then become the same form as those of the above model:

$$
\begin{array}{r}
\epsilon=\frac{4 M_{\mathrm{PL}}^{2}}{3 \xi_{1}^{2} h^{4}} \\
\eta=-\frac{4 M_{\mathrm{PL}}^{2}}{3 \xi_{1} h^{2}} .
\end{array}
$$

From the COBE normalization $\frac{\lambda_{e f f}}{\xi_{1}^{2}} \sim 10^{-10}$. This model was arguing the requirement of non-minimal coupling $\left(\xi_{1}\right)$ of Higgs sector with the curvature tensor. The coupling of SM Higgs with the four-form plays a crucial role in this scenario as well. This model $\left[\lambda_{e f f}=\frac{\lambda}{4}\right]$ of inflation is questionable because it may violate unitarity before reaching the inflationary scale [9 12]. If we address the inflation with an effective self-coupling constant, it is possible to reduce the value of non-minimal coupling constant.

\section{B. Minimal Higgs Inflation with the Non-Minimal Coupling of Tensor Field with} gravity

We have the freedom to choose different combinations of couplings with the gravity. If we switch off $\xi_{1}\left(\xi_{1}=0\right)$, then $\xi_{\text {eff }}=2 C_{1} \xi_{2}$. We can then obtain another inflationary scenario from this kind of coupling without changing the values of any other inflation parameters. The inflaton potential is given by

$$
U(\phi)=\frac{M_{\mathrm{PL}}^{4} \lambda_{e f f}}{\left(2 C_{1} \xi_{2}\right)^{2}}\left[\frac{\left(\exp \left(\sqrt{\frac{2}{3}} \frac{\phi}{M_{\mathrm{PL}}}\right)-\left(1+\frac{2 \xi_{2} q_{1}}{M_{\mathrm{PL}}^{2}}\right)\right)^{2}}{\exp \left(2 \sqrt{\frac{2}{3}} \frac{\phi}{M_{\mathrm{PL}}}\right)}\right],
$$

where $\lambda_{\text {eff }}=\left(\frac{\lambda}{4}+\frac{C_{1}^{2}}{2}\right)$. The slow-roll parameters for this scenario can be obtained

$$
\begin{aligned}
& \epsilon=\frac{4\left(M_{\mathrm{PL}}^{2}+2 \xi_{2} q_{1}\right)^{2}}{3\left(2 C_{1} \xi_{2}\right)^{2} h^{4}} \\
& \eta=\left(\frac{4}{3}\right) \frac{\left(M_{\mathrm{PL}}^{2}+2 \xi_{2} q_{1}\right)\left(M_{\mathrm{PL}}^{2}+2 \xi_{2} q_{1}-\left(2 C_{1} \xi_{2}\right) h^{2}\right)}{\left(2 C_{1} \xi_{2}\right)^{2} h^{4}} .
\end{aligned}
$$


The values for $n_{s}$ and $r$ can be calculated using the formalism that we discussed in the previous subsection. They preserve their values because of the form of the potential. For $\mathcal{N}=60$ the calculated values for $n_{s}$ and $r$ equal $n_{s}=0.9633$ and $r=0.0032$ with $\lambda_{\text {eff }}=$

$\left(\frac{\lambda}{4}+\frac{C_{1}^{2}}{2}\right)$ and $\xi_{\text {eff }}=2 C_{1} \xi_{2}$. Using the COBE results $\frac{\lambda_{\text {eff }}}{\left(C_{1} \xi_{2}\right)^{2}} \sim 10^{-10}$. Another feature of this model is that the SM Higgs field is free from the non-minimal coupling of gravity.

\section{CONCLUSIONS}

In this work, we have addressed the inflation in the framework of the SM with the presence of 4-form interactions. Compared to the existing models of Higgs inflation, we have modified the values of $\lambda$ and $\xi$ in the presence of 4 -form-flux couplings. We also see that the four-form interactions can generate the Higgs-boson mass before electroweak symmetry breaking and such generated Higgs mass is also quantized. It is suggested that the 4-form flux plays an important role in this inflationary scenario. By switching off the components of $\xi_{\text {eff }}$, we can re-obtain the minimal and non-minimal Higgs inflation scenarios.

We have introduced the most general Lagrangian for the inflation models. We have also realized that there is no need for the Higgs-gravity coupling to produce the right amount of density perturbation. In Ref. [6] the authors discussed about the requirement of the Higgs-gravity coupling in order to give a successful inflation model. However, we have shown explicitly that it is possible to address the inflation without the Higgs non-minimal coupling. We can reproduce the results of Ref. [6] from our model.

\section{ACKNOWLEDGMENT}

This work was supported by the Taiwan Ministry of Science and Technology under Grant No. MOST-107-2112-M-007-029-MY3.

[1] G. F. Giudice, A. Kehagias and A. Riotto, "The Selfish Higgs," JHEP 1910, 199 (2019) doi:10.1007/JHEP10(2019)199 [arXiv:1907.05370 [hep-ph]].

[2] N. Kaloper and A. Westphal, "A Goldilocks Higgs," arXiv:1907.05837 [hep-th]. 
[3] N. Kaloper and A. Lawrence, "Natural chaotic inflation and ultraviolet sensitivity," Phys. Rev. D 90, no. 2, 023506 (2014) doi:10.1103/PhysRevD.90.023506 [arXiv:1404.2912 [hep-th]]. E. Dudas, "Three-form multiplet and Inflation," JHEP 1412, 014 (2014) doi:10.1007/JHEP12(2014)014 [arXiv:1407.5688 [hep-th]].

[4] H. M. Lee, "Chaotic inflation with four-form couplings," Phys. Rev. D 101, no. 4, 043523 (2020) doi:10.1103/PhysRevD.101.043523 [arXiv:1908.05475 [hep-ph]].

[5] H. M. Lee, "Relaxation of Higgs mass and cosmological constant with four-form fluxes and reheating," JHEP 2001, 045 (2020) doi:10.1007/JHEP01(2020)045 arXiv:1908.04252 [hep$\mathrm{ph}]$.

[6] F. L. Bezrukov and M. Shaposhnikov, "The Standard Model Higgs boson as the inflaton," Phys. Lett. B 659, 703 (2008) doi:10.1016/j.physletb.2007.11.072 [arXiv:0710.3755 [hep-th]].

[7] D. I. Kaiser, "Conformal Transformations with Multiple Scalar Fields," Phys. Rev. D 81, 084044 (2010) doi:10.1103/PhysRevD.81.084044 [arXiv:1003.1159 [gr-qc]].

[8] Y. Akrami et al. [Planck Collaboration], "Planck 2018 results. X. Constraints on inflation," arXiv:1807.06211 [astro-ph.CO].

[9] C. P. Burgess, H. M. Lee and M. Trott, "Power-counting and the Validity of the Classical Approximation During Inflation,” JHEP 0909, 103 (2009) doi:10.1088/1126-6708/2009/09/103 arXiv:0902.4465 [hep-ph]].

[10] M. Atkins and X. Calmet, "Remarks on Higgs Inflation," Phys. Lett. B 697, 37 (2011) doi:10.1016/j.physletb.2011.01.028 arXiv:1011.4179 [hep-ph]].

[11] J. Ren, Z. Z. Xianyu and H. J. He, JCAP 1406, 032 (2014) doi:10.1088/14757516/2014/06/032 [arXiv:1404.4627 [gr-qc]].

[12] Z. Z. Xianyu, J. Ren and H. J. He, Phys. Rev. D 88, 096013 (2013) doi:10.1103/PhysRevD.88.096013 [arXiv:1305.0251 [hep-ph]]. 